\title{
Relativistic Corrections to the Triton Binding Energy
}

\author{
F. Sammarruca, D. P. Xu', and R. Machleidt \\ Department of Physics, University of Idaho, \\ Moscow, Idaho 83843, U.S.A.
}

September 5, 2018

\begin{abstract}
The influence of relativity on the triton binding energy is investigated. The relativistic three-dimensional version of the Bethe-Salpeter equation proposed by Blankenbecler and Sugar (BbS) is used. Relativistic (non-separable) one-boson-exchange potentials (constructed in the BbS framework) are employed for the two-nucleon interaction. In a 34-channel Faddeev calculation, it is found that relativistic effects increase the triton binding energy by about $0.2 \mathrm{MeV}$. Including charge-dependence (besides relativity), the final triton binding energy predictions are 8.33 and $8.16 \mathrm{MeV}$ for the Bonn $\mathrm{A}$ and $\mathrm{B}$ potential, respectively.
\end{abstract}

$\dagger$ Present address: Department of Physics, Massachusetts Institute of Technology, Cambridge, Massachusetts 02139. 


\section{Introduction}

One of the main objectives of nuclear few-body physics is to learn something about nuclear forces. When the few-body problem can be solved accurately, results can be related directly to the elementary (two- and many-body) forces used as input in the calculations. Since the two-nucleon scattering data determine the nucleon-nucleon (NN) interaction only on-shell, one has to consider A-nucleon systems with $A>2$ to test off-shell properties of nuclear potentials. Similarly, many-body forces can only be tested in systems with $A>2$. Guided by these ideas, comprehensive work has been performed for, particularly, the trinucleon $(3 N)$ system. For this system, an exact and practically feasible theory has been developed by Faddeev [1].

In recent years, many benchmark calculations of the triton binding energy employing realistic $N N$ potentials have been performed [2, 3, 4] — with the objective of obtaining (indirect) information on the off-shell behaviour of the two-nucleon potential and to assess the size of the nuclear three-body force. It has been shown that when two-nucleon forces only are applied, the triton is underbound by an amount which varies from 0.2 to $1.1 \mathrm{MeV}$ (depending on which NN potential is used in the calculation). The conventional interpre- 
tation of this result is that it 'proves empirically' the existence of three-body forces (which would contribute $0.2 \mathrm{MeV}$ or more to the triton binding). As shown in many calculations [5], it is then possible to patch up the discrepancy with experiment by including phenomenological three-body forces which can easily be fit to any amount of missing binding energy. Unfortunately, not much physics can be learned in this ad hoc fashion.

Strictly speaking, most many-body forces are an artefact of theory. They are created by freezing out degrees of freedom contained in the full Hamiltonian of the problem under consideration. This fact suggests how one may learn some physics from three-body-force calculations: when a new degree of freedom is introduced (besides the nucleon), it should be taken into account consistently in the two- and the three-body problem. The theory of the NN interaction has shown that isobar degrees of freedom (particularly the $\Delta(1232))$ are crucial for a realistic and reliable description of the nuclear force [6]. Trinucleon calculations taking the $\Delta$-degree of freedom consistently into account have been performed first by the Hannover group [7]. Recently, this type of calculation has been updated and improved [8]. The new result is that the sum of all three-body force contributions arising from the $\Delta$-isobar is repulsive; the repulsive contribution to the triton binding energy 
varies between 0.06 and $0.4 \mathrm{MeV}$ depending on the $\Delta$ model [8]. This small total result is due to a cancelation between an attractive three-body force contribution of about $1.5 \mathrm{MeV}$ and a slightly larger repulsive dispersive effect on the $\Delta$-diagrams contained in the two-nucleon interaction. Even if the $\pi-N S$-wave part of a $2 \pi$-exchange three-body force [9] (not included in a $\Delta$ model) is added, the total result is essentially vanishing. Thus, there is little hope that a consistent theory for two- and three-body forces will explain the triton binding energy within the conventional framework.

On the background of the above discussion, it becomes increasingly important to investigate aspects left out in conventional three-nucleon calculations. One of these aspects is relativity. Most present calculations using realistic two-body forces are performed in a non-relativistic framework. Thus, one may ask whether relativistic effects are responsible for the missing binding. Since the triton wave functions contain high momentum components, it is by no means clear that the non-relativistic approximation is justified. Furthermore, some non-relativistic predictions for the triton binding energy (obtained with two-body forces only) are only $0.2 \mathrm{MeV}$ off the empirical value 国. Thus, even if relativistic effects are small, they may be large as compared to the small gap to be filled by 'non-conventional' nuclear physics. 
In summary, the current status of the triton binding energy program calls for an accurate knowledge of the relativistic corrections.

In the past 25 years, there have been numerous efforts to estimate relativistic effects in the three-nucleon system. Roughly, one can distinguish between two lines of research. In a model independent approach, one starts from the Poincaré algebra for the ten generators of the Poincaré group, expressed in terms of the degrees of freedom of $n$ particles only. This frame work is applied in Refs.[10-13] and critically examined in Refs.[14-16].

An alternative approach is to use a field-theoretic framework and the Bethe-Salpeter equation [17] or one of its three-dimensional reductions. Pioneering work along this line has been performed by Tjon and coworkers [18, 19]. In reference [19], a Blankenbecler-Sugar [20] three-body equation for a modified Reid potential is solved. In a series of recent studies, Rupp and Tjon solved the full four-dimensional three-body Bethe-Salpeter equation 21, 22]. However, to make this tremendous numerical project feasible, finite-rank separable NN potentials had to be used. In these studies, relativistic effects are found to increase the triton binding by about $0.3 \mathrm{MeV}$.

In the present paper, we investigate the influence of relativity in the $3 N$ bound- state system using the relativistic meson-theoretic Bonn poten- 
tial [6, 23]. It is important that relativistic few-body calculations are performed consistently. By this we mean that the same relativistic formalism has to be used for the two- and the three-body systems. Since the relativistic potential we apply is constructed in the framework of the Blankenbecler-Sugar equation [20] (a relativistic three-dimensional reduction of the Bethe-Salpeter equation [17]), we use an equivalent relativistic equation for the three-nucleon system.

Various three-dimensional relativistic three-body equations have been proposed, which all have in common the elimination of the relative time component in the original four-dimensional integration over internal momenta. This is usually done by replacing, in the three-particle Bethe-Salpeter equation, the original Green's function by one that involves a delta function. This procedure is carried out in such a way as to preserve relativistic invariance, three-particle unitarity and, of course, the correct non-relativistic limit.

As mentioned above, our study is performed within such relativistic threedimensional approaches, combining, however, a non-separable relativistic two-body potential (the Bonn potential), with relativistic Faddeev equations. Among our objectives it is to understand to which extent, if at all, the type of two-body potential used is related to the size of the relativistic effect. Finally, 
our main motivation is that the influence of relativity on the $3 N$ bound-state properties is a basic and fundamental problem, and we must achieve a solid understanding of it.

In the next Section, we will briefly recall the relevant equations and describe the relativistic corrections. In Section 3 we present and discuss our results.

\section{Formalism}

The relativistic two-body problem is described by the Bethe-Salpeter (BS) [17] equation, which can be extended to the three-body case. Similarly to the non-relativistic formalism, the relativistic three-body BS equation can be cast into Faddeev form [20, 24, 25, 26]. Unlike in the non-relativistic case, however, one obtains a four-dimensional integral equation, (the additional integration variable is due to the intermediate particles being off their mass shell), which drastically complicates the numerical solution. To avoid this difficulty, various three-dimensional reductions have been worked out as a practical generalization of the relativistic Faddeev equations. Here, we just briefly recall the procedure. The relativistic Faddeev equations are written 
as

$$
T^{i}=t_{i}+\sum_{j \neq i} t_{i} G_{0 i} T^{j}
$$

with $i, j=1,2,3 . t_{i}$ are two-body scattering operators describing all interactions between particles $j$ and $k$, and $G_{0 i}$ is the free two-particle Green's function of the pair $j, k$ acting in the three-body space. (In the non-relativistic case, it can be shown that $G_{01}=G_{02}=G_{03}$, all particles being on their mass shell.) The relativistic propagator, e.g. for particles $j$ and $k$, is then

$$
G_{0 i}=\frac{1}{\left(k_{j}^{2}-m^{2}+i \epsilon\right)} \frac{1}{\left(k_{k}^{2}-m^{2}+i \epsilon\right)}
$$

with $k_{i}$ the four-momentum of particle $i$ in the three-body center-of-mass (C.M.) frame. The usual procedure is then to replace the relativistic fourdimensional propagators by a three-dimensional one which is derived from a dispersion integral [18, 19]. This amounts to introducing a Green's function which produces the proper right-hand (unitarity) cut. The procedure is, however, not unique, and many different versions can be found in the literature.

Instead of the Green's function Eq. (2), one may write a dispersion integral in terms of the two-particle invariant energy, $\sigma_{i}$, (as we choose to do in 
this work), namely

$$
g_{i}=4 \int_{4 m^{2}}^{\infty} \frac{d \sigma_{i}^{\prime}}{\sigma_{i}^{\prime}-\sigma_{i}} \delta\left[\left(P_{j k}^{\prime} / 2+k_{j k}\right)^{2}-m^{2}\right] \times \delta\left[\left(P_{j k}^{\prime} / 2-k_{j k}\right)^{2}-m^{2}\right]
$$

where $k_{j k} \equiv\left(k_{j}-k_{k}\right) / 2, \sigma_{i}=\left(P-k_{i}\right)^{2}=P_{j k}^{2}$ with $P=k_{i}+k_{j}+k_{k}$ the total four-momentum, $P_{j k} \equiv k_{j}+k_{k}$, and $P_{j k}^{\prime}=\sqrt{\sigma_{i}^{\prime} / \sigma_{i}} P_{j k}$. In the three-body C.M. frame, $P=(\sqrt{s}, \mathbf{0})$ with $\sqrt{s}=3 m-E_{b}$ the invariant mass of the three-body system ( $m$ denotes the mass of a free nucleon and $E_{b}$ the binding energy of the three-nucleon system). Clearly, this choice cannot be unique, since any function which does not contribute to the right-hand cut of $\sigma$ can be added without affecting unitarity. Evaluation of the integral in Eq. (3) results in the three-dimensional propagator [18, 19]

$$
g=\frac{4}{\omega_{p}} \frac{1}{4 \omega_{p}^{2}+|\mathbf{q}|^{2}-\left(\sqrt{s}-\omega_{q}\right)^{2}}
$$

Here, $\mathbf{q}\left(\mathbf{q}^{\prime}\right)$ are the momenta of the spectator particle in the initial(final) state, respectively, $\omega_{p}=\sqrt{m^{2}+|\mathbf{p}|^{2}}, \mathbf{p}=-\frac{1}{2} \mathbf{q}-\mathbf{q}^{\prime}$, and $\omega_{q}=\sqrt{m^{2}+|\mathbf{q}|^{2}}$. Notice that this is just a more convenient notation which allows to drop particles indices, namely $\mathbf{q}=\mathbf{k}_{i}, \mathbf{q}^{\prime}=\mathbf{k}_{k}^{\prime}$, where $i$ and $k$ are understood to be the spectator particles in the initial and final state respectively. Clearly, $\mathbf{q}$ and $\mathbf{q}^{\prime}$ are also the relative momenta between the spectator and the interacting pair in the three-body C.M., see Fig. 1. 
Alternatively, one could have written the dispersion integral in terms of the three-particle invariant energy, that is

$$
g_{i}=4 \int_{9 m^{2}}^{\infty} \frac{d s^{\prime}}{s^{\prime}-s} \delta\left\{\left[\left(P^{\prime}-k_{i}\right) / 2+k_{j k}\right]^{2}-m^{2}\right\} \times \delta\left\{\left[\left(P^{\prime}-k_{i}\right) / 2-k_{j k}\right]^{2}-m^{2}\right\}
$$

with $k_{j k}$ as above, $s=P^{2}, P^{\prime}=P \sqrt{s^{\prime} / s}$. The resulting three-dimensional propagator would then be 18,21$]$

$$
g=\frac{2}{\omega_{1} \omega_{2}} \frac{\omega_{1}+\omega_{2}+\omega_{3}}{\left(\omega_{1}+\omega_{2}+\omega_{3}\right)^{2}-s}
$$

with $\omega_{1}=\sqrt{m^{2}+\left|\mathbf{q}^{\prime}\right|^{2}}, \omega_{2}=\sqrt{m^{2}+\left|\mathbf{q}+\mathbf{q}^{\prime}\right|^{2}}$ and $\omega_{3}=\sqrt{m^{2}+|\mathbf{q}|^{2}}$.

Other alternatives have also been suggested. One could write as well the dispersion integral in terms of the one-particle invariant energy, or introduce relativistic kinematics in the free resolvent of the non-relativistic Faddeev equation; in the latter case, phase space factors are introduced to make the volume element of the integration relativistically invariant [18]. All choices are equivalent in the sense that they satisfy three-body unitarity and preserve the correct non-relativistic limit. This non-relativistic Faddeev propagator reads in our notation

$$
g_{N R}=\frac{1}{m} \frac{1}{|\mathbf{p}|^{2}+\frac{3}{4}|\mathbf{q}|^{2}+m E_{b}}
$$

Besides the modifications in the propagator, additional corrections must 
be included to properly define the dynamical variables. Clearly, in the threebody calculation, these are defined in the overall C.M. system and must appear correctly in the input two-body t-matrix. The diagram in Fig. 1 is a typical graphical representation of the three-particle reaction $i(j k) \rightarrow$ $k(i j)$. The double lines represent interacting pairs (with momenta $-\mathbf{q},-\mathbf{q}^{\prime}$, respectively). The total invariant mass of the two-body sub-system is

$$
\sigma=\left(\sqrt{s}-E_{q}\right)^{2}-|\mathbf{q}|^{2}
$$

with $E_{q}=\sqrt{m^{2}+|\mathbf{q}|^{2}}$, so that $\left(\sqrt{s}-E_{q}\right)$ is nothing but the energy of the pair with total three-momentum $\mathbf{- q}$ (righthandside of diagram in Fig. 1).

Also, the relative momenta of the interacting pair must be defined in a relativistic way (in a non-relativistic theory, they would be the Galilean invariant relative momenta). It has been shown shown [26, 27 that the relativistic relative momentum, (which reduces to the momentum of one of the particles in their C.M. system), is given by the combination

$$
\mathbf{Q}=\mathbf{q}+\rho\left(|\mathbf{q}|,\left|\mathbf{q}^{\prime}\right|, z\right) \mathbf{q}^{\prime}
$$

(for the lower vertex in Fig. 1). In the last expression, $z$ is the angle between 
$\mathbf{q}$ and $\mathbf{q}^{\prime}$, and the function $\rho$ is given by [26, 27]

$$
\rho\left(|\mathbf{q}|,\left|\mathbf{q}^{\prime}\right|, z\right)=\xi_{q^{\prime}}^{-1 / 2}\left(E_{q}+\frac{\mathbf{q} \cdot \mathbf{q}^{\prime}}{\xi_{q^{\prime}}^{1 / 2}+E_{q}+E_{\mathbf{q}+\mathbf{q}^{\prime}}}\right)
$$

with $\xi_{q^{\prime}}=\left(E_{q}+E_{\mathbf{q}+\mathbf{q}^{\prime}}\right)-\mathbf{q}^{\prime 2}$. (A completely analogous definition applies to the upper vertex, but with $\mathbf{q}, \mathbf{q}^{\prime}$ interchanged.) Notice that $\mathbf{Q}, \mathbf{Q}^{\prime}$ are formally identical to the Galilean invariant relative momenta, if the function $\rho$ is replaced by a factor $1 / 2$.

Finally, we mention that the relativistic two-body t-matrix, $t_{r e l}$, is related to the non-relativistic one, $t_{N R}$, by

$$
t_{r e l}=t_{N R} \sqrt{E / m} \sqrt{E^{\prime} / m}
$$

with $E=\sqrt{m^{2}+\mathbf{Q}^{2}}$ and $E^{\prime}=\sqrt{m^{2}+\mathbf{Q}^{\prime 2}}$. If in the relativistic BlankenbeclerSugar $(\mathrm{BbS})$ equation, $t_{r e l}$ is replaced by Eq. (11) and the quasi-potential, $V_{r e l}$, is replaced by

$$
V_{\text {rel }}=V_{N R} \sqrt{E / m} \sqrt{E^{\prime} / m}
$$

then the usual non-relativistic Lippmann-Schwinger equation is obtained [28].

In summary, the relativistic calculation of the triton binding energy differs from the non-relativistic one in three points:

1. The relativistic Faddeev propagator, Eq. (4), is used instead of the conventional non-relativistic propagator, Eq. (7). 
2. The kinematical variables of the two interacting nucleons are defined in a covariant way (cf. Eqs. (8) and (9)).

3. An invariant two-body t-matrix is used, cf. Eq. (11).

It will turn out that the last point (invariance of the t-matrix) has the largest quantitative effect and is responsible for the increase of the triton binding energy.

\section{Results and Discussion}

In Table 1 we present our results for the non-relativistic and relativistic calculations of the triton binding energy taking 5 and 34 three-body channels [29] into account. We apply the Bonn A and the Bonn B potential [30] for the two-body interaction. The relativistic calculations are based upon the formalism outlined in the previous section, with the three-nucleon propagator given in Eq. (4). For the solution of the non-relativistic as well as relativistic Faddeev equation, we use standard momentum-space techniques [31].

We find a relativistic correction to the triton binding energy of $0.19 \mathrm{MeV}$

for the Bonn A and $0.20 \mathrm{MeV}$ for the Bonn B potential. As indicated above, 
the largest effect comes from the use of an invariant t-matrix; this increases the binding energy by $0.26 \mathrm{MeV}$. The effect of using covariant kinematical variables is very small and further increases the binding energy by $0.07 \mathrm{MeV}$, while the relativistic Faddeev propagator, Eq. (4), reduces the binding energy by $0.14 \mathrm{MeV}$. (The numbers given here for the partial effects refer to the 34channel calculation with Bonn A.)

Using the Bonn A potential, the non-relativistic 34-channel result of 8.32 $\mathrm{MeV}$ is increased to 8.51 by relativity. These numbers refer to the chargeindependent calculation in which the neutron-proton potential is used for all states. If the well-established charge-dependence in the ${ }^{1} S_{0}$ two-nucleon potential is taken into account, the triton binding energy is reduced by 0.18 $\mathrm{MeV}$, yielding the final result of $8.33 \mathrm{MeV}$ for the Bonn A potential. For Bonn $\mathrm{B}$, the final result, including relativistic effects and charge-dependence in the ${ }^{1} S_{0}$ state, is $8.16 \mathrm{MeV}$ (cf. Table 1 ). In our calculations, charge dependence is taken into account as described in Ref. [32].

The relativistic effect we find is small but not negligible. Another subtle effect that is usually neglected in triton binding energy calculations is charge-dependence. Accidentally this effect is of the same magnitude as the relativistic effect. For this reason, we also consider charge-dependence in 
our calculations. Thus, we can provide rather 'complete' final results for the triton binding energy predictions from the two-body force only. This final result is that the triton is underbound by $0.15-0.30 \mathrm{MeV}$ depending on the choice of the relativistic two-nucleon potential. This means that these potentials almost completely explain the triton binding energy in terms of just the two-nucleon force.

At this point, one may raise the question whether it is reasonable to obtain almost all the triton binding energy from the two-body force alone. The crucial issue here is what to expect from three-body forces. As mentioned in the Introduction, the recent investigations by Picklesimer et al. [8] have confirmed the earlier findings by the Hannover group [7] that a consistent treatment of two- and three-body forces results in an almost vanishing total contribution to the triton binding from three-body forces. In this light, the triton binding can only be understood as resulting essentially from the two-nucleon interaction (unless one wants to invoke subhadronic degrees of freedom).

Thus, crucial for the understanding of our result is the nature of the two-body force. It is well-known [23] that a weaker tensor force component in the two-nucleon force implies more attraction in few- and many-body 
systems. This is why Bonn A predicts more binding than Bonn B; and, even more so, this is the reason why both Bonn A and B give substantially more binding energy than other conventional potentials which, in general, predict only about $7.5 \mathrm{MeV}$. On the other hand, the tensor force should only be as weak as allowed by the requirement that the potential be 'realistic'. The usual interpretation of the term 'realistic' is that the potential should reproduce the two-nucleon scattering data up to pion-production threshold. This is, in fact, true for both Bonn A and B, with the exception of the $\epsilon_{1}$ mixing parameter at energies larger than $100 \mathrm{MeV}$, which is insufficiently reproduced by Bonn A, but correctly predicted by Bonn B [33]. On those grounds, one may be tempted to cast some doubt on the Bonn A three-body result.

To properly discuss this issue, let us first focus on the Bonn B predictions. The Bonn B potential is with no doubt realistic (in its predictions of all two-body bound state and scattering observables, including the $\epsilon_{1}$ ), and nevertheless predicts substantially more triton binding than other realistic potentials. For example, the Paris and the Bonn B potential yield almost identical predictions for phase shifts, $\epsilon$ parameters, and observables [34], i. e. they are as identical on-shell as two different potentials can be. However, 
the triton binding energy prediction by Bonn $\mathrm{B}$ is about $0.4 \mathrm{MeV}$ larger than the one by Paris (the charge-dependent Paris result is $7.59 \mathrm{MeV}$ [3]). This clearly reveals off-shell differences between the two interactions. The Paris potential is, apart from a $\mathbf{p}^{2}$ operator, a local potential, while Bonn A and $\mathrm{B}$ are defined in terms of the relativistic momentum-space Feynman amplitudes for one-boson-exchanges (which are clearly non-local expressions). These off-shell differences can already be seen in the D-state probability of the deuteron which is $5.8 \%$ for Paris and $5.0 \%$ for Bonn B.

Concerning now the Bonn A potential, one has to understand the relevance of the $\epsilon_{1}$ parameter for nuclear ground state predictions. In Reference [35], a family of potentials has been considered that has the same low D-state probability of the deuteron as Bonn A, but larger $\epsilon_{1}$ parameter at intermediate energies. It is found that the triton binding energy is always the same and, thus, is not affected by the behaviour of the $\epsilon_{1}$ above $100 \mathrm{MeV}$.

Finally, some comments are in place concerning the relativistic framework we use. Given the fact that our relativistic one-boson-exchange two-nucleon potentials are constructed within the BbS scheme, the relativistic three-body equation derived within the $\mathrm{BbS}$ framework certainly is the most consistent choice. 
On the other hand, for the relativistic three-nucleon problem, (unlike the non-relativistic case), predictions from state-of-the-art calculations do not corroborate and are, in fact, quite method-dependent. For instance, Rupp and Tjon have shown 21, 22 that the BbS approximation yields about $75 \%$ of the relativistic effect as obtained from the four-dimensional BS equation. The reason for this difference may be the effective three-body forces due to retardation which are automatically included in the four-dimensional BS equation and do not exist in static approaches. 21, 22]

Naïvely, one would believe that the BS equation is a priori the 'best' equation. Based upon this believe, the quality of a relativistic three-dimensional equation would then be determined by how close it reproduces results obtained from the BS equation.

However, as pointed out by Gross [36], there are physical reasons why this seemingly most plausible approach may not be correct. For example, the BS equation in ladder approximation (that is, with a kernel which is restricted to one-boson-exchanges) does not generate the desired one-body equation in the one-body limit (i. e. when one of the particles becomes very massive), while a large family of three-dimensional relativistic reductions of the BS equation does have the correct one-body limit. If the sum of all crossed 
ladders is added to the ladders, then the correct limit is obtained in the BS equation. As a matter of fact, in almost all applications in nuclear physics (including the present work) the kernel is restricted to one-boson-exchanges. Furthermore, when going beyond the second order kernel, there is no rapidly converging series of irreducible kernels in the BS framework [36]. Better convergence is obtained for some three-dimensional equations.

Thus, the BS equation may not be the optimal choice for relativistic investigations in nuclear physics. Given the problems intrinsic to relativistic approaches, three-dimensional relativistic equations and results obtained from them may be equally meaningful or even superior as compared to the four-dimensional BS theory.

The authors are indebted to R. A. Brandenburg for his momentum-space Faddeev code. This work was supported in part by the NSF-Idaho EPSCoR program under Grant No. RII-8902065, the NSF Physics program under Grant No. PHY-8911040, and by the San Diego Supercomputer Center.

\section{References}

[1] L. D. Faddeev, Soviet Physics. JETP 12, 1041 (1961). 
[2] E. P. Harper, Y. E. Kim, and A. Tubis, Phys. Rev. C 6, 126 (1972);

A. Laverne and C. Gignoux, Nucl. Phys. A203, 597 (1973);

R. A. Brandenburg, Y. E. Kim, and A. Tubis, Phys. Rev. C 12, 1368 $(1975)$

R. A. Brandenburg, P. U. Sauer, and R. Machleidt, Z. Physik A280, 93 (1977);

Ch. Hajduk and P. U. Sauer, Nucl. Phys. A369, 321 (1981);

C. R. Chen, G. L. Payne, J. L. Friar, and B. F. Gibson, Phys. Rev. C 31, $2266(1985)$;

T. Sasakawa and S. Ishikawa, Few-Body Systems 1, 3 (1986).

[3] H. Witala, W. Glöckle, and H. Kamada, Phys. Rev. C 43, 1619 (1991).

[4] R. A. Brandenburg, G. S. Chulick, R. Machleidt, A. Picklesimer, and R. M. Thaler, Phys. Rev. C 37, 1245 (1988).

[5] A. Bömelberg, Phys. Rev. C 34, 14 (1986);

C. R. Chen, G. L. Payne, J. L. Friar, and B. F. Gibson, Phys. Rev. C 33, $1740(1986)$;

S. Ishikawa and T Sasakawa, Few-Body Systems 1, 143 (1986). 
[6] R. Machleidt, K. Holinde, and Ch. Elster, Phys. Reports 149, 1 (1987).

[7] Ch. Hajduk, P. U. Sauer, and W. Strueve, Nucl. Phys. A405, 581 (1983); M. T. Peña, H. Henning, and P. U. Sauer, Phys. Rev. C 42, 885 (1990).

[8] A. Picklesimer, R. A. Rice, and R. Brandenburg, Phys. Rev. C 44, 1359 (1991); ibid. 45, 547 (1992); ibid. 45, 2045 (1992).

[9] J. L. Friar, B. F. Gibson, G. L. Payne, and S. A. Coon, Few-Body Systems 5, 13 (1988).

[10] P. A. Dirac, Rev. Mod. Phys. 21, 392 (1949); B. Bamhanjian and L. H. Thomas, Phys. Rev. 92, 1300 (1953); S. N. Sokolov, Docl. Akad. Nauk USSR, 233, 575 (1977); F. Coester and W. N. Polyzou, Phys. Rev. D 26, 1348 (1982).

[11] L. L. Foldy and R. A. Krajcik, Phys. Rev. Lett. 32, 1025, (1974); Phys. Rev. D 12, 1700 (1975).

[12] V. K. Gupta, B. S. Bhahar, and A. N. Mitra, Phys. Rev. Lett. 15, 974 (1965); V. S. Bhasin, H. Jacob, and A. N. Nitra, Phys. Lett. 32B, 15 (1970); H. Jacob, V. S. Bhasin, and A. N. Mitra, Phys. Rev. D 1, 3496 (1970). 
[13] L. A. Kondratyuk, F. M. Lev, and V. V. Soloview, Few-Body Systems 7 55 (1989).

[14] L. Müller, Nucl. Phys. A360, 331 (1981).

[15] W. Glöckle, T. S. H. Lee, and F. Coester, Phys. Rev. C 33, 709 (1986).

[16] F. Coester, Helv. Phys. Acta 38, 7 (1965).

[17] E. E. Salpeter and H. A. Bethe, Phys. Rev. 84, 1232 (1951).

[18] A. Ahmadzadeh and J. A. Tjon, Phys. Rev. 147, 1111 (1966).

[19] A. D. Jackson and J. A. Tjon, Phys. Lett. 32B, 9 (1970).

[20] R. Blankenbecler and R. Sugar, Phys. Rev. 142, 1051 (1966).

[21] G. Rupp and J. A. Tjon, Phys. Rev. C 37, 1729 (1988).

[22] G. Rupp and J. A. Tjon, Phys. Rev. C 45, 2133 (1992).

[23] R. Machleidt, Adv. Nucl. Phys. 19, 189 (1989).

[24] V. A. Alessandrini and R. L. Omnes, Phys. Rev. 139, B167 (1965).

[25] D. Z. Freedman, C. Lovelace, and J. M. Namyslowsky, Nuovo Cimento 43A, 258 (1966). 
[26] R. Aaron, R. D. Amado, and J. E. Young, Phys. Rev. 174, 2022 (1968).

[27] A. S. Rinat and A. W. Thomas, Nucl. Phys. A282, 365 (1977).

[28] For more details, see Section 4.1 of Ref. [27].

[29] For a definition of the three-body channels see, e. g., Ref. 四.

[30] The Bonn A and B potentials are presented in Section 4 and Appendix A, Table A.1, of Ref. [23].

[31] W. Glöckle and R. Offermann, Phys. Rev. C 16, 2039 (1977); W. Glöckle, G. Hasberg, and A. R. Neghabian, Z. Physik A305, 217 (1982).

[32] R. A. Brandenburg, G. S. Chulick, Y. E. Kim, D. J. Klepacki, R. Machleidt, A. Picklesimer, and R. M. Thaler, Phys. Rev. C 37, 781 (1988).

[33] See Fig. 4.4 of Ref. [23].

[34] See Table 5.2 and Figs. 5.10-12 of Ref. [23] where phase parameters and observables as predicted by the Paris and the Bonn B potential are shown. (Note that the Bonn B potential is denoted by 'OBEP' in the cited Table and Figures, while 'Bonn' denotes the Bonn full model of Ref. [6].) 
[35] R. Machleidt, XII. Int. Conf. on Few-Body Problems in Physics, Vancouver, B. C., Canada, 1989, Contributed Papers, B. K. Jennings, ed. (TRIUMF Report TRI-89-2) p. G51; R. Machleidt et al., submitted to Few-Body Systems.

[36] F. Gross, Phys. Rev. C 26, 2203 (1982). 
Table 1: Triton binding energy (in units of $\mathrm{MeV}$ ) obtained in non-relativistic and relativistic calculations using two versions of the relativistic Bonn twonucleon potential.

\begin{tabular}{c|c|c|c|c}
\hline \hline Potential & $\begin{array}{c}\text { Number } \\
\text { of } \\
\text { Channels }\end{array}$ & Non-Relativistic & Relativistic & $\begin{array}{c}\text { Relativistic } \\
\text { and } \\
\text { Charge-Dependent }\end{array}$ \\
\hline \hline Bonn A & 5 & 8.37 & 8.53 & 8.35 \\
& 34 & 8.32 & 8.51 & 8.33 \\
\hline Bonn B & 5 & 8.16 & 8.33 & 8.15 \\
& 34 & 8.14 & 8.34 & 8.16 \\
\hline \hline
\end{tabular}


Figure 1: Graphical representation of the three-body rearrangement collision $i(j k) \rightarrow k(i j)$. Double lines represent interacting pairs. The underlying time axis is horizontal, pointing left into the future. 